\title{
Risk Factors, and Clinical and Etiological Characteristics of Ischemic Strokes in COVID-19-Infected Patients: A Systematic Review of Literature
}

\author{
Simone Vidale $\mathrm{e}^{\mathrm{a}, \mathrm{b}}$ \\ aDepartment of Neurology, Infermi Hospital, Rimini, Italy; ${ }^{\text {b}}$ Department of Neuroscience, Ospedale Moriggia \\ Pelascini, Gravedona, Italy,
}

\section{Keywords}

Coronavirus disease 2019 · Stroke · Etiology

\begin{abstract}
Background and Purpose: Coronavirus disease 2019 (COVID-19) infection is an ongoing pandemic and worldwide health emergency that has caused important changes in healthcare systems. Previous studies reported an increased risk of thromboembolic events, including stroke. This systematic review aims to describe the clinical features and etiological characteristics of ischemic stroke patients with COVID-19 infection. Method: A literature search was performed in principal databases for studies and case reports containing data concerning risk factors, clinical features, and etiological characteristics of patients infected with COVID-19 and suffering from stroke. Descriptive and analytical statistics were applied. Results: Overall, 14 articles were included for a total of 93 patients. Median age was 65 (IQR: 55-75) years with prevalence in males. Stroke occurred after a median of 6 days from COVID-19 infection diagnosis. Median National of Institute of Health Stroke Scale (NIHSS) score was 19. Cryptogenic (Cry) strokes were more frequent (51.8\%), followed by cardioembolic etiology, and they occurred a
\end{abstract}

long time after COVID-19 diagnosis compared with large-artery atherosclerosis strokes ( $p_{\text {trend: }} 0.03$ ). The clinical severity of stroke was significantly associated with the severity grade of COVID-19 infection ( $p_{\text {trend: }}$ 0.03). Conclusions: Ischemic strokes in COVID-19-infected patients were clinically severe, affecting younger patients mainly with Cry and cardioembolic etiologies. Further multicenter prospective registries are needed to better describe the causal association and the effect of COVID-19 infection on stroke.

(C) 2021 S. Karger AG, Basel

\section{Introduction}

The new coronavirus disease 2019 (COVID-19) infection is an ongoing pandemic and worldwide health emergency that has influenced healthcare systems since the first report in Wuhan, China, in December 2019. This virus has been associated with an increased risk of acute thromboembolic events, including pulmonary embolism, myocardial infarction, and stroke [1]. For this last disease, some reports published in the literature described cases of patients affected by COVID-19, which developed stroke symptoms. In one of the first retrospective studies

$\begin{aligned} & \text { karger@karger.com } \\ & \text { www.karger.com/ced }\end{aligned}$
Karger ${ }^{\prime /}$

Simone Vidale

Department of Neurology, Infermi Hospital

Viale Luigi Settembrini, 2

IT-47923 Rimini (Italy)

simone.vidale@auslromagna.it 
Table 1. Demographic and clinical characteristics of included patients

\begin{tabular}{lc}
\hline & Value \\
\hline Age, years (median and IQR) & $65(55-75)$ \\
Gender (males)* & $62(66.7 \%)$ \\
NIHSS (median and IQR) & $19(12-21)$ \\
Days between COVID-19 diagnosis and stroke & $6(2-11)$ \\
\hline
\end{tabular}

NIHSS, National of Institute of Health Stroke Scale; COVID-19, coronavirus disease $2019 .{ }^{*}$ Five patients with missing data.

of hospitalized patients from Wuhan, about $5.7 \%$ of the severe patients with the infection was complicated by a stroke [2]. This review of the literature describes the main risk factors, clinical features, and etiological characteristics of patients infected with COVID-19 and suffering from a stroke.

\section{Method}

The author systematically searched PubMed, Embase, and Cochrane Central register of Controlled Trials for studies containing information concerning risk factors, and clinical and etiological features of patients with ischemic stroke and infected with $\mathrm{CO}$ VID-19 published between December 2019 and June 25, 2020. The search strategy included the combination of terms, such as "cerebrovascular disorder," "stroke," "COVID-19," "coronavirus," and "SARS-CoV2," as either keywords or MeSH terms. Reference lists and citing articles were also reviewed to increase the identification of relevant studies. Retrospective and prospective studies and case reports were included in this review. The language of the included studies was limited to English. In this review, demographic data concerning age and gender were introduced. Vascular risk factors reported by studies in this systematic review were hypertension, diabetes mellitus, dyslipidemia, atrial fibrillation, and coronary artery disease. The clinical severity at admission or at stroke onset was summarized by the National of Institute of Health Stroke Scale (NIHSS), considering mild, moderate, moderate to severe, and severe stroke as a score of $<6,6-15,16-20$, and $>21$, respectively. The etiology was classified using the TOAST criteria, considering large-artery atherosclerosis (LAA), cardioembolism (CE), smallvessel disease, and lacunar (Lac), cryptogenic (Cry), and other causes of stroke. Finally, the days between the COVID-19 diagnosis and stroke occurrence were reported, as well as the clinical severity of COVID-19 infection. Studies or case reports that did not include some of the previous data were excluded from the systematic analysis. In the statistical analysis, the continuous and categorical variables were presented as median (IQR) and $n$ (\%), respectively. The Mann-Whitney $U, \chi^{2}$, Fisher exact, and ANOVA tests were used to compare differences between groups of patients. A $p<0.05$ was considered statistically significant. Statistical analysis were done using SPSS software.
Table 2. Distribution of vascular risk factors and etiological subgroups

\begin{tabular}{lc}
\hline & Value $n(\%)$ \\
\hline Vascular risk factors & \\
Missing data & $11(11.8)$ \\
No risk factors & $17(20.7)$ \\
Hypertension & $47(57.3)$ \\
Diabetes mellitus & $28(34.1)$ \\
Dyslpidemia & $27(32.9)$ \\
Atrial fibrillation & $14(17.1)$ \\
Coronary artery disease & $18(22)$ \\
Etiological categories & \\
Missing data & $10(20.8)$ \\
LAA & $12(14.5)$ \\
CE & $22(26.5)$ \\
Lac & $4(4.8)$ \\
Cry & $43(51.8)$ \\
Oth & $2(2.4)$ \\
\hline
\end{tabular}

$\%$ for vascular risk factors and etiological categories are reported in relation to available data. LAA, large-artery atherosclerosis; CE, cardioembolism; Lac, lacunar; Cry, cryptogenic; Oth, other causes of stroke.

\section{Results}

Overall, 14 articles were included in this analysis for a total of 93 patients [3-15]. The flowchart of the literature search was reported in see online suppl. Material; see www.karger.com/doi/10.1159/000514247 for all online suppl. material. The median age was 65 (IQR: 55-75) years, with prevalence in males ( $n: 62 ; 70.5 \%$ of patients with available data). Stroke occurred after a median of 6 days from COVID-19 diagnosis (IQR: 2-11 days), and more than half of the patients were severely ill from COVID-19 infection. The median NIHSS score was 19 (IQR: $12-21$ ), with a prevalence of moderate to severe strokes, as reported in Table 1.

In 17 patients, no vascular risk factors were reported, while the main risk factor was represented by hypertension $(n: 47 ; 57.3 \%$ of patients with available data), followed by diabetes mellitus. The distribution of each considered risk factor and missing data are represented in the Table 2.

Considering the etiological categories, in 10 patients, no data were reported (10.8\%). In the remaining cases, Cry strokes represented the main etiology, reported in about half the patients $(n: 43,51.8 \%)$. CE was the second etiological category, accounting for 22 cases $(26.5 \%)$. The entire distribution of etiological categories is represented in Table 2. 
A significant association was observed between the etiological classification and the interval between the COVID-19 diagnosis and the cerebrovascular event ( $p_{\text {trend: }}$ 0.039 ). Cry strokes occurred mainly in longer temporal intervals after COVID-19 diagnosis with a mean of 9.1 days of delay. This time was significantly higher in LAA (1.7-day lag) or Lac strokes (4.7-day lag). Cardioembolic strokes occurred with a mean of 5.1-day lag. The graphic of these differences is given in online suppl. material. Similarly, a significant association was observed between etiological classification and age ( $p: 0.02)$. Cry strokes were represented mainly by younger patients (mean age: 62 years), while the elderly were affected principally by $\mathrm{CE}$ (mean age: 72.7 years). Box plot of the distribution of age means by etiological categories is reported in online suppl. material. Finally, the clinical severity of stroke was significantly associated with the severity grade of $\mathrm{CO}$ VID-19 infection ( $p_{\text {trend: }}$ 0.03).

\section{Discussion}

This systematic review concerning clinical and etiological features of ischemic strokes in COVID-19-infected patients is reported for the first time in the literature that acute cerebrovascular events occurred in younger subjects with mainly a Cry etiology and moderate to severe clinical severity. This profile differs from "classical" epidemiological data concerning ischemic strokes. In particular, previous studies provided results with a lower rate of Cry strokes and a higher number of patients with Lac strokes [16]. If younger age is considered, a more weak difference between the results of this review and those of previous studies was observed, with reported Cry stroke rates varying from 21.9 to $36.7 \%[17,18]$, and this is in line with the observed association between etiological subgroups and age. All these findings could be related to new pathological mechanisms that are caused by the COVID-19 infection. In particular, some previous studies showed that new coronavirus induces a hypercoagulated status, determining elevated levels of D-dimer and fibrinogen $[19,20]$. Another possible mechanism is represented by the endothelial dysfunction due to depletion angiotensin-converting enzyme 2 . In this condition, angiotensin II could not be counterbalanced by angiotensin-converting enzyme 2 , producing a pro-inflammatory state, other than vasoconstriction, and promoting a tissue injury also in the brain and their vessels [20]. In light of these hypotheses, Lac and LAA strokes should be less represented in COVID-19 patients affected by acute cerebro-

Ischemic Strokes in COVID-19-Infected Patients vascular events at the expense of Cry and cardioembolic strokes, as reported in this review. All the previous pathological mechanisms could not be immediate, and a worsening organ injury or prothrombotic status contribute in few days to the thromboembolic events, as observed in this review with a mean temporal delay of 8 days between COVID-19 diagnosis and cardioembolic strokes. These different etiological patterns also imply preventive therapeutic strategies that include an higher use of anticoagulant treatments, supporting the recommendations for a prompt prophylactic anticoagulation with low-molecular weight heparin [21]. However, immediate therapy with anticoagulants should be balanced with the risk of intracranial hemorrhage, and for this reason, clinical trials are warranted to evaluate the treatment efficacy and safety.

Considering the clinical severity, the included patients presented severe symptoms. This observation could also be influenced by the fact that mild neurological symptoms were not recognized or detected in COVID-19-infected patients.

A limitation of this review is the partial description of COVID-19-related ischemic strokes due to no reported quota of patients in the literature. A second limitation is due to about $10 \%$ missing data for some variables, but it is in line with other studies and systematic reviews. Finally, the sources of data acquisition are all represented by retrospective studies or case series/case reports, limiting the homogeneity and the completeness of information. In conclusion, this systematic review shows peculiar etiological and clinical features concerning acute ischemic strokes in patients infected with COVID-19, but in the future, multicenter prospective registries are needed to better characterize the causal association and the clinical pattern of new cases.

\section{Conflict of Interest Statement}

The author declares there is no conflict of interest to declare.

\section{Funding Sources}

The author did not receive any funding. 


\section{References}

1 Klok FA, Kruip MJHA, van der Meer NJM, Arbous MS, Gommers DAMPJ, Kant KM, et al. Incidence of thrombotic complications in critically ill ICU patients with COVID-19. Thromb Res. 2020 Jul;191:145-7.

2 Mao L, Wang M, Chen S, He Q, Chang J, Hong C, et al. Neurological manifestations of hospitalized patients with COVID-19 in Wuhan, China: a retrospective case series study. SSRN Electron J. 2020.

3 Zhou F, Yu T, Du R, Fan G, Liu Y, Liu Z, et al. Clinical course and risk factors for mortality of adult in patients with COVID-19 in Wuhan, Chine: a retrospective cohort study. Lancet. 2020;395:1054-62.

4 Zayet S, Klopfenstein T, Kovacs R. Acute cerebral stroke with multiple infarctions and COVID-19. France; 2020.

5 Wang A, Mandingo GK, Yim PD, Meyers PM, Lavine SD. Stroke and mechanical thrombectomy in ptients with COVID-19: technical observations and patient characteristics. J Neurointerv Surg. 2020 Jul;12(7):64853.

6 Gunasekaran K, Amoah K, Rajasurya V, Buscher MG. Stroke in a young COVID-19 patient. QJM. 2020 May 22;113(8):573.

7 Oxley TJ, Mocco J, Majidi S, Kellner CP, Shoirah H, Singh IP, et al. Large-vessel stroke as a presenting feature of Covid-19 in the young. N Engl J Med. 2020;382(20):e60.
8 Yaghi S, Ishida K, Torres J, Mac Grory B, Raz E, Humbert K, et al. SARS-CoV-2 and stroke in a New York healthcare system. Stroke. 2020 Jul;51(7):2002-11.

9 Co COC, Yu JRT, Laxamana LC, David-Ona DIA. Intavenous thrombolysis for stroke in a COVID-19 positive Filipino patient, a case report. J Clin Neurosc. 2020 Jul;77:234-6.

10 Tunç A, Unlubas Y, Alemdar M, AkyÜz E. Coexistence of COVID-19 and acute ischemic stroke report of four cases. J Clin Neurosc. 2020 Jul;77:227-9.

11 Avula A, Nalleballe K, Narula N, Sapozhnikov S, Dandu V, Toom S, et al. COVID-19 presenting as stroke. Brain Behav Immun. 2020 Jul;87:115-9.

12 Beyrouti R, Adams ME, Benjamin L, Cohen H, Farmer SF, Goh YY, et al. Characteristics of ischaemic stroke associated with COVID-19. J Neurol Neurosurg Psychiatry. 2020 Aug;91(8):889-91.

13 D’Anna L, Kwan J, Brown Z, Halse O, Jamil S, Kalladka D, et al. Characteristics and clinical course of Covid-19 patients admitted with acute stroke. J Neurol. 2020 Nov;267(11): 3161-65.

14 Escalard S, Maier B, Redjem H, Delvoye F, Hébert S, Smajda S, et al. Treatment of acute ischemic stroke due to large vessel occlusion with COVID-19. Stroke. 2020 Aug;51(8): 2540-43.
15 Morassi M, Bagatto D, Cobelli M, D’Agostini S, Gigli GL, Bnà C, et al. Stroke patients with SARS-CoV-2 infection: case series. J Neurol. 2020 Aug;267(8):2185-192.

16 McArdle PF, Kittner SJ, Ay H, Brown RD Jr, Meschia JF, Rundek T, et al. Agreement between TOAST and CCS ischemic stroke classification. The NINDS SiGN Study. Neurology. 2014;83:1653-60.

17 Fromm A, Haaland ØA, Naess H, Thomassen L, Waje-Andreassen U. Atherosclerosis in trial of org 10172 in acute stroke treatment subtypes among young and middle-aged stroke patients: the Norwegian stroke in the young study. J Stroke Cerebrovasc Dis. 2016;25(4): 825-30.

18 Chatzikonstantinou A, Wolf ME, Hennerici MG. Ischemic stroke in young adults: classification and risk factors. J Neurol. 2012;259(4): 653-9.

19 Tang N, Bai H, Chen X, Gong J, Li D, Sun Z. Anticoagulant treatment is associated with decreased mortality in severe coronavirus disease 2019 patients with coagulopathy. J Thromb Haemost. 2020 May;18(5):1094-9.

20 Hess DC, Eldahshan W, Rutkowski E. COVID-19-related stroke. Transl Stroke Res. 2020; 11(3):322-5.

21 Thachil J, Tang N, Gando S, Falanga A, Cattaneo M, Levi M, et al. ISTH interim guidance on recognition and management of coagulopathy in COVID-19. J Thromb Haemost. 2020 May;18(5):1023-6. 